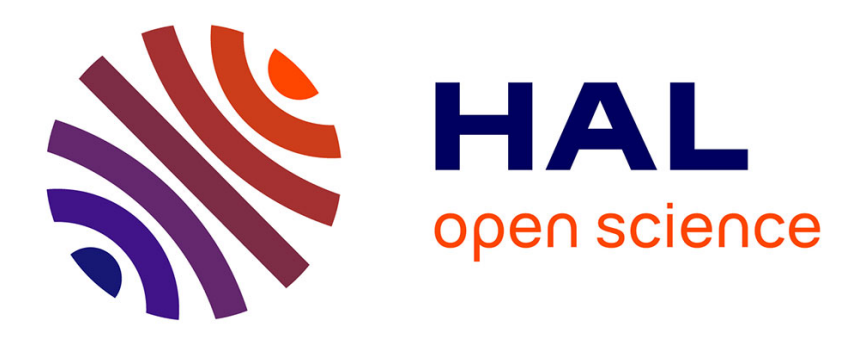

\title{
Photographie aérienne et problèmes forestiers
}

Paul Rey

\section{To cite this version:}

Paul Rey. Photographie aérienne et problèmes forestiers. Revue forestière française, 1953, 11, pp.735-

745. 10.4267/2042/26945. hal-03384175

\section{HAL Id: hal-03384175 \\ https://hal.science/hal-03384175}

Submitted on 18 Oct 2021

HAL is a multi-disciplinary open access archive for the deposit and dissemination of scientific research documents, whether they are published or not. The documents may come from teaching and research institutions in France or abroad, or from public or private research centers.
L'archive ouverte pluridisciplinaire HAL, est destinée au dépôt et à la diffusion de documents scientifiques de niveau recherche, publiés ou non, émanant des établissements d'enseignement et de recherche français ou étrangers, des laboratoires publics ou privés. 


\section{PHOTOGRAPHIE AERIENNE ET PROBLEMES FORESTIERS}

La photographie aérienne a ses lettres de noblesse: des résultats spectaculaires ont été obtenus au cours de ces dernières années dans les domaines de l'archéologie, des sciences humaines, de la prospection géologique, de l'inventaire des forêts américaines, et d'une manière plus générale, dans la recherche du renseignement. De tels succès ont attiré l'attention de tous, techniciens et chercheurs, sur cette remarquable source de documentation dont on avait primitivement apprécié la seule application cartographique, qui n'est pas la moindre, mais exige des moyens d'exécution puissants, apanage d'organismes hautement spécialisés comme l'Institut Géographique National.

Sous l'impulsion de l'I.G.N., des collections importantes de photographies sont aujourd'hui disponibles en France, pour la totalité du territoire, et en de nombreuses régions de l'Union Française. Un instrument de travail d'une efficacité remarquable est ainsi mis à la disposition d'un nombre croissant d'utilisateurs, l'exploitation des photographies aériennes étant possible par des méthodes simples, n'exigeant qu'une spécialisation minime et un matériel réduit.

C'est grâce à ces méthodes que, dès I945, le Service de la Carte de la Végétation de la France a pu largement développer l'interprétation photographique des paysages végétaux qui constitue désormais la base des travaux de cartographie botanique et écologique entrepris en France et Outre-Mer.

Des stages d'interprétation photographique ont pu être organisés par le Centre National de la Recherche Scientifique en $195^{2}$ et I953, en liaison avec diverses administrations et plus particulièrement l'Administration forestière. De fructueux échanges de vures ont permis, au cours de ces stages, de mettre les méthodes d'interprétation photographique à l'épreuve de la réalité technique et de jeter les bases d'une Introduction à la Photogrammétrie Forestière, fruit d'une collaboration étroite entre techniciens et utilisateurs de la photographie: nous sommes heureux de les remercier ici pour le précieux concours qu'ils nous ont apporté.

Les pages qui suivent ont pour objet de préciser quelques applications de ces méthodes de recherche à l'étude du milieu naturel, et plus précisément à la connaissance, au recensement et à la mise en valeur des richesses végétales du territoire. Elles supposent con- 
nues les notions de photogrammétrie élémentaire indispensables, dont unq analyse pratique a été faite d'autre part, à l'occasion d'une communication, en avril 1953, au $78^{\mathrm{e}}$ Congrès des Sociétés Savantes, à Toulouse.

Il convient cependant de souligner avant toute chose l'importance de la précision de l'observation; en effet, la précision des méthodes photogrammétriques doit être garantie par l'identification rigoureuse sur les photographies des points sur lesquels s'appuient les constructions nécessaires. C'est en cela que les travaux graphiques à partir des photographies diffèrent profondément des travaux exécutés à partir de cartes ou de plans: dans ce dernier cas, il n'y a aucune difficulté au cours d'une même opération, à utiliser sans ambiguité les points de référence choisis initialement: tout report de mesures d'angles ou de distances pourra être fait sans risque d'erreur. Pour qu'il en soit de même dans une étude photogrammétrique, il est indispensable que l'attention de l'opérateur soit constamment soutenue et que son interprétation ne varie pas an cours de l'opération: il prendra grand soin, par exemple, à toujours considérer le même angle d'un carrefour ou le même détail du terrain choisi comme point de référence; la plupart des erreurs ou imprécisions dans les constructions opérées proviennent de la nonobservation de ces règles.

\section{I. - Principes d'identification félímentairl:}

\section{Orientation}

I.a lecture d'une photographie sera plus aisée, surtout sous le stéréoscope, si l'on prend soin d'orienter le document de telle sorte que les ombres soient dirigées vers l'observateur: on acquerra ainsi une impression de relief plus nette, souvent même perceptible sans le secours du stéréoscope, sous une forte loupe par exemple.

\section{$2^{\circ}$ Critères d'identification}

La photographie aérienne a, sur l'observation au sol, le grand avantage de donner une vue d'ensemble et de fixer dans le temps et l'espace les rapports entre les divers éléments du paysage. Chaque détail pouvant être étudié dans son cadre, l'interprétation doit être axée à la fois sur l'étude des caractères propres à chacun d'eux et sur leur comportement dans l'ensemble de la photographie.

En d'autres termes, l'identification élémentaire doit être basée sur deux groupes de critères:

- des critères photogrammétriques: examen et mesure de la partie visible de l'élément à étudier; 
- dos critères d'cnvironnement tirés de l'examen de l'ensemble de la photographie considéré dans ses rapports avec l'élément étudié.

\section{$3^{\circ}$ Examen $d u$ (" toit )}

Ia partic visible de l'élément à étudier constitue le " toit » (" ronf " des auteurs de langue anglaise). Ce toit est défini par sa forme, sa structure et son relief: on ne fera appel aux notions cle teinte qu'avec une grande prudence, car elles peuvent varier pour diverses causes : émulsion du cliché, éclairement, conditions stationnelles, saisons, etc... (un même résineux pourra, par exemple, paraitre plus sombre ou plus clair, suivant les cas, qu'un feuillu voi$\sin )$.

Ces caractéristiques du toit se traduisent sur l'image photographique par les aspects souvent très révélateurs dus essentiellement à des jeux de lumière, d'ombres et d'ombres portées, celles-ci étant plus intenses que les ombres normales. Tous les problèmes d'identification peuvent se ramener à la recherche des contrastes et à leur analyse en fonction $\mathrm{du}$ comportement de la lumière par rapport au toit.

\section{$4^{\circ}$ Phases de l'interprétation}

L'interprétation photographique ne dispense jamais d'opérations au sol. mais ces opérations doivent être menées en fonction de l'utilisation des photographies; dans la grande généralité des cas. on procédcra suivant les stades suivants:

a) esquissce photogrammétrique préparatoire:

b) opération de renseignement au sol suivant un itinéraire 01 des points précisés par le premier travail;

c) étude photogrammétrique définitive;

d) contrôle par sondages au sol.

\section{II. - ORDREs D'IDENTIFICATION ÉLÍMENTATRE}

I es renseignements susceptibles d'être recueillis par interprétation photogrammétrique peuvent être groupés en cinq ordres:

- l'identification physionomique;

- l'identification spécifique;

- l'identification stationnelle;

- l'identification spatiale;

- l'identification historique.

Au point de vue forestier ou botanique, ces cinq ordres peuvent être définis de la façon suivante: 


\section{$1^{\circ}$ Identification physionomique ou structurale}

Elle a pour objet de rechercher:

a) la structure physionomique des éléments du tapis végétal niaturel ou transformé: forêts, landes, pelouses, cultures, plantations, etc... (son application est alors d'ordre essentiellement cartographique).

b) la structure des peuplements dans le cadre d'une étude d'aménagement ou d'inventaire forestier.

L'investigation physionomique permet de supprimer presque totalement les opérations au sol de recensement et de cartographie des limites.

On pourra, suivant l'objet des recherches et la région considérée, établir des tableaux d'identification physionomique, tels que celui qui est reproduit ci-après, relatif aux principales formations végétales rencontrées en France:

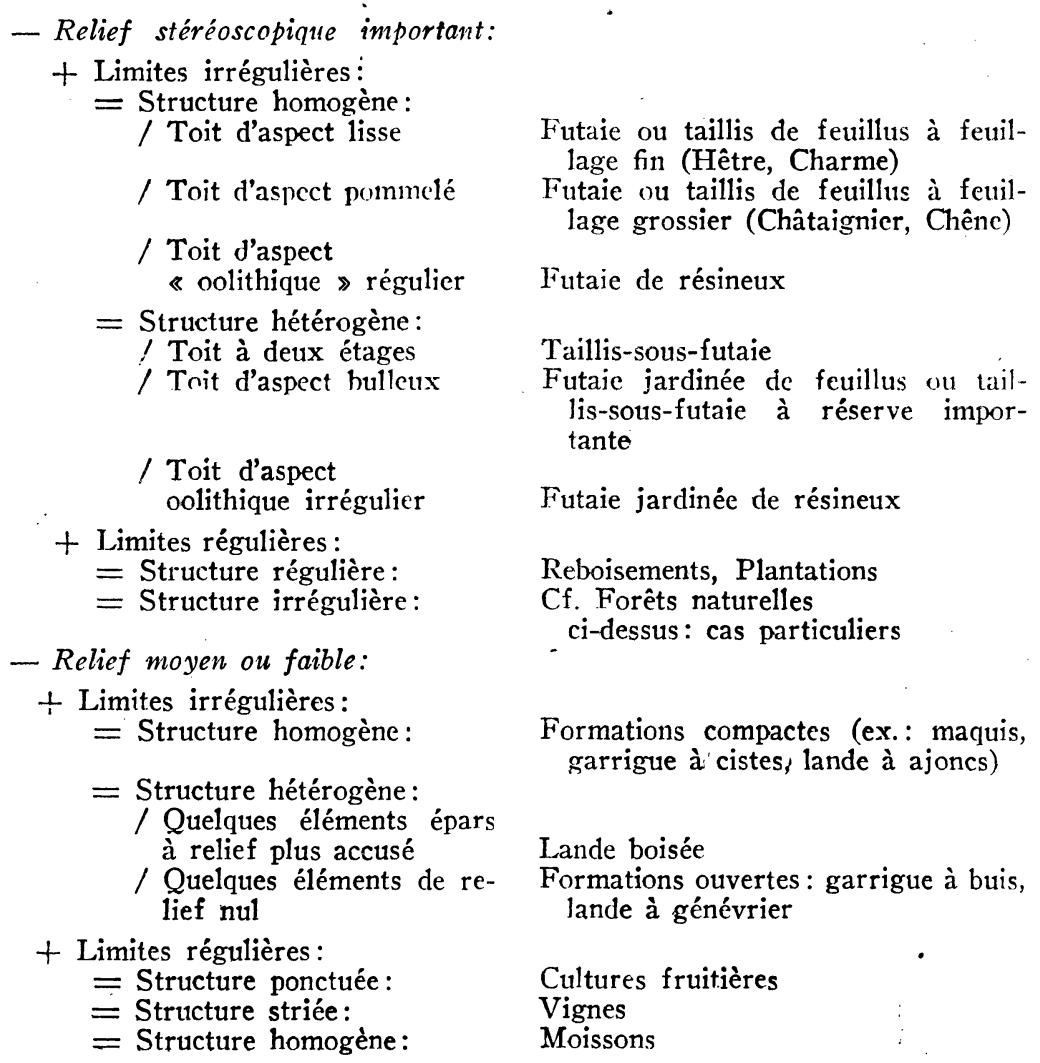




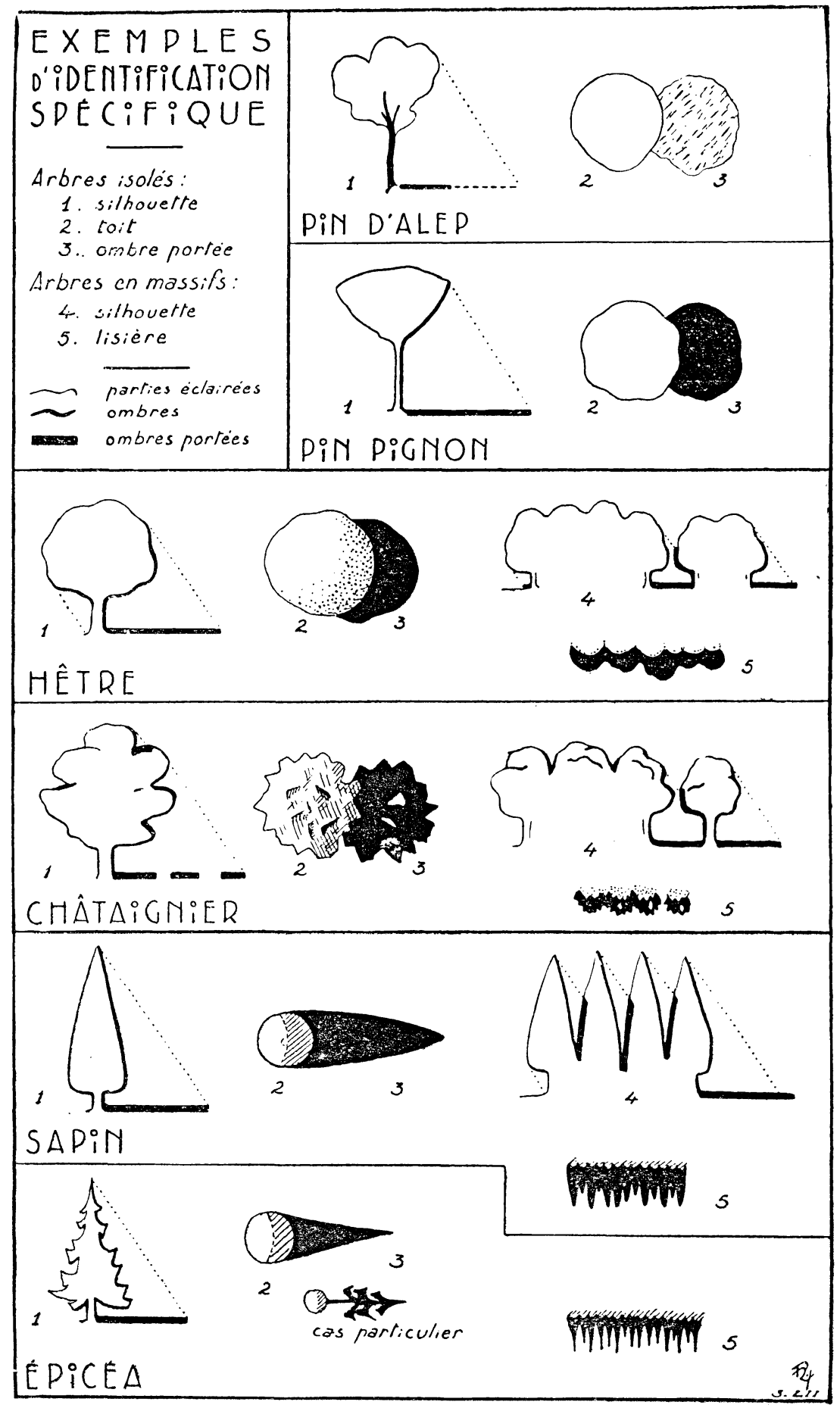

Voir Stéréogrammes au verso

IDENTIFICATION DE LA VÉGÉTATION PAR LA PHOTOGRAPHIE AÉRIENNS.

(Extrait du Bulletin de la Société d'Histoirc Naturelle de Toulouse, T. 87, $\Upsilon_{952}$, P1. I.) 

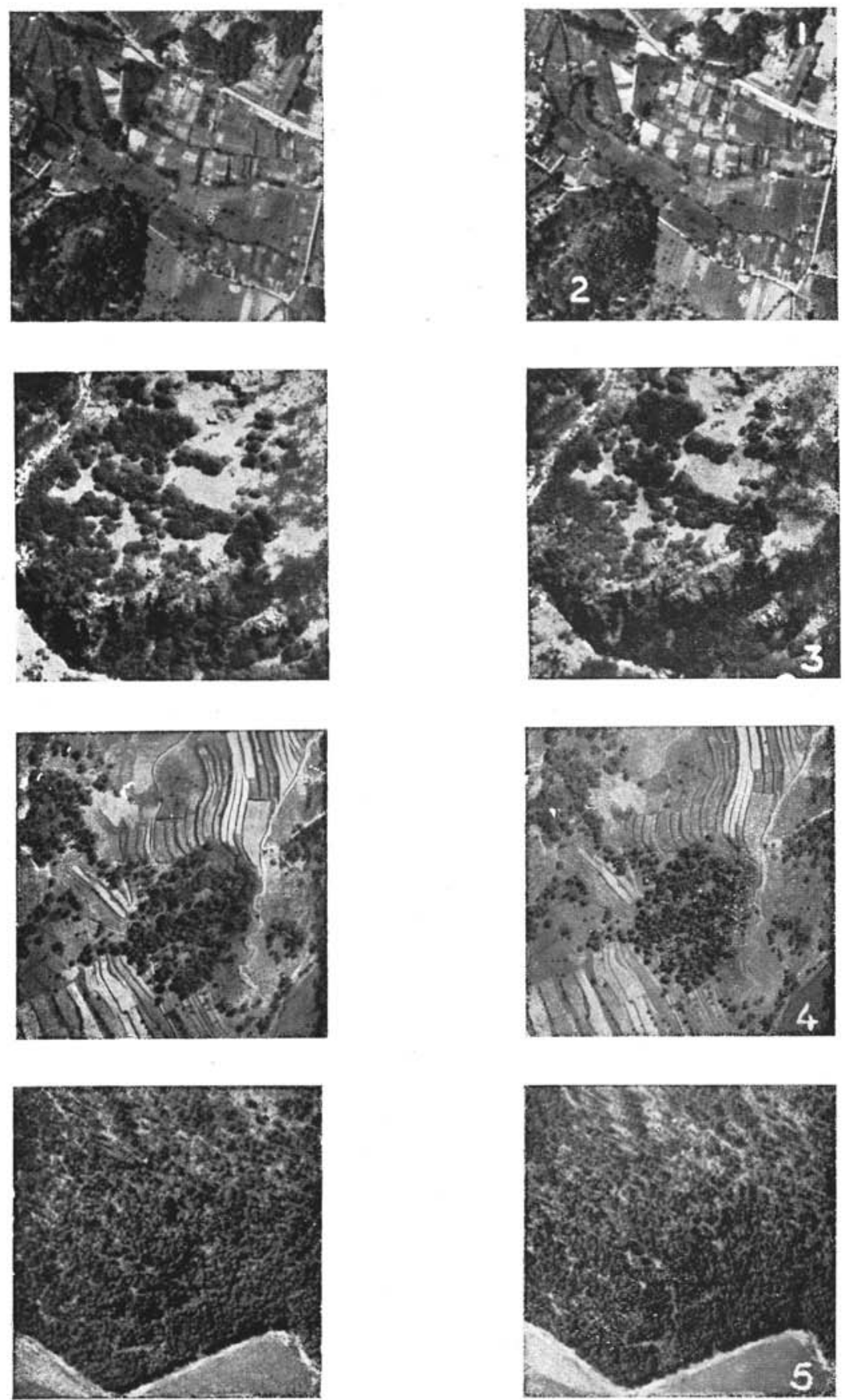

1-2. Pin d'Alep et Pin Pignon. - 3. Hêtre. - 4. Châtaignier. -- 5. Sapin. (Clichés Armée américaine - Phototheque Inst. géograph. national Echelle I/I2 000 - I/I5 000.)

Inentification de la végétation par ta photogirapilie á́ktinne. (Extrait du Bulletin de la Société d'Histoire Naturelle de Toulouse, T. 87, I952, Pl. II.) 
- Relief nul:

+ Limites irrégulières:

$=$ Teinte claire, aspect moiré

$=$ Teinte sombre

$=$ Teinte très sombre homogène

= Eléments épars à relief accusé

Pclouse sèche

Pelouse humide, tourbière

Prairie naturelle

Pré - bois

+ Limites régulières:

$=$ Structure finement striée

teintes diverses

$=$ Structure homogène

teinte sombre

Cultures, labours

Prairie artificielle

\section{$2^{\circ}$ Identification spécifique}

La détermination photographique des essences forestières doit être basée sur:

a) les caractéristiques de l'image photographique de chaque essence à l'état isolé et en massif ; aspect général; dimensions et forme de la couronne; dimensions, forme et compacité de l'ombre et de l'ombre portée (critères photogrammétriques). Ces caractéristiques de l'image doivent être interprétées en fonction des caractères: propres de l'arbre; port, transparence du feuillage, nature de l'ombre, etc... ;

b) les conditions stationnelles (critères d'environnement) et la connaissance des exigences écologiques des diverses essences;

c) la probabilité de détection de telle ou telle essence dans un secteur donné.

La précision de l'identification spécifique est fonction de la qualité et de l'échelle de la photographie, l'échelle idéale étant de I/ Io o00I/I 5000 .

Dans la plupart des cas, l'investigation spécifique doit être liée à des opérations au sol, mais il est cependant possible à un observateur entraîné de faire des déterminations certaines, uniquement par examen de photographies.

Les planches ci-contre illustrent cette méthode de détermination $\left(^{*}\right)$; elles concernent des ( couples ) d'essences forestières dont l'iclentification comparée est particulièrement instructive :

Pin d'Alep: toit à contour imprécis, transparent; sol visible; ombre imprécise; impression de "flou » en massif.

Pin pignon: toit circulaire, à contour précis, homogène; impression d'un disque épais posé sur le sol; ombre compacte détachée de l'arbre.

(*) La reproduction en similigravure altérant la netteté des photographies, certains détails caractéristiques sur les documents originaux sont devenus pratiquement invisibles sur les stéréogrammes imprimés planche II. 
Hêtre : isolé: toit arrondi, homogène, sans rugosité; ombre très compacte, circulaire, détachée de l'arbre; - en massif: toit à aspect spongieux très caractéristique, dî̀ à la transparence du feuillage en surface (donc à l'absence d'ombres entre arbres rapprochés) et à la compacité d'ensemble du feuillage (donc fortes ombres portées aux interruptions du toit); lisière ondulante.

Châtaignier : isolé: toit arrondi dans l'ensemble, anguleux dans ie détail, à rugosités apparentes ; ombre anguleuse, parfois trouée ; - en massif: arbres individualisés, plantations régulières' fréquentes.

Sapin: isolé: toit très éclairé latéralement ; impression de « point trouble ) au sommet, dû au fait que la pellicule n'enregistre pas la cime au delà de $90 \mathrm{~cm}$ de diamètre pour un cliché au I/I2 000 ; ombre très compacte à contour convexe, non détachée de l'arbre ; - en massif: aspect granuleux " oolithique » ; lisière caractéristique.

Epicéa: caractères analogues, mais ombres plus effilées, à contour concave; lisière caractéristique.

\section{$3^{\circ}$ Identification stationnelle}

La photographie aérienne est particulièrement précieuse pour éclairer les conditions de " site ) des objets. L'observation stéréoscopique, souvent mieux que l'observation au sol, permet d'étudier dans son cadre un peuplement forestier ou un groupement végétal: altitude, pente, exposition, nature du sol, conditions d'humidité nu de drainage, érosions, etc..., peuvent être immédiatement décelées : l'interprétation stationnelle est un puissant adjuvant de l'identification spécifique, et constitue dans beaucoup de cas l'intérêt majeur de l'utilisation des photographies.

\section{$4^{\circ}$ Identification spatiale}

Dans cette rubrique peuvent être groupées toutes les mesures faites sur photographies: distances, longueurs, hauteurs, surfaces; on pcut y ajouter les évaluations de densité et de volume, pour lesquelles il sera utile de disposer de tables de références (Cf. infra III, $3^{\circ}$ ).

Les diverses méthodes photogrammétriques pourront être appliquées ici suivant le cas, mais il faut noter que cette identification est la seule qui requiert parfois un matériel spécialisé: loupe micrométrique, barre parallaxe, éventuellement stéréocomparagraphe.

La précision des mesures variera donc en fonction du matériel disponible, de la méthode utilisée et aussi de l'échelle des photographies. L'erreur principale sera toujours imputable à la mesure elle-même : dans des conditions normales d'observation, la hauteur et les dimensions d'un arbre isolé ou en lisière d'un massif. peuvent être appré- 
ciées avec une erreur de 0,50 à $2 \mathrm{~m}$. S'il s'agit d'arbres en massif dense, l'erreur peut être supérieure, car il sera nécessaire d'apprécier les mesures relativement aux arbres de lisière (périphérie, layons. ou clairières): on ne peut alors raisonnablement envisager que la mesure de la hauteur moyenne des arbres.

Il faut tenir compte en outre, dans toute mesure ou tout comptagc (évaluation de dencité), du fait qu'à l'échelle (lu I/ I 5 ooo, tout oljjct de dimension inférieure à 0,90 est invisible sur la photographic.

\section{$5^{\circ}$ Identification historique}

L'identification historique groupe enfin toutes les possibilités d'exploitation des photographies dans l'étude de phénomènes " historiques ) impliquant:

- soit la connaissance instantanée d'un événement fortuit: enneigement, inondation, avalanche, incendie, attaque parasitaire, etc...;

- soit la mise en évidence d'événements échelonnés dans le temps: coupes, reboisements, travaux d'équipement, etc...

Cettc identification historique est particulièrement fructueuse lorsqu'on dispose pour une même région, de collections de photographies successives.

\section{IIT. - J'IDENTIFICATION COMPLEXE}

L'investigation photographique peut revêtir une fornie plus complexe et faire appel à la fois à toutes les formes d'identification élémentaire définies ci-dessus. C'est sous cette forme synthétique quielle sera généralement le plus efficace, mais cela suppose une grande pratique d'observation et la connaissance de tous les éléments de photogrammétrie ou d'interprétation exposés plus haut.

L’identification complexe a pour objet. devant un problème précis, clont les éléments d'information au sol sont à priori limités, cle définir sur les photographies des zones d'égale v'aleur dans le cadre du problème considéré.

Ces zones d'égale valeur - ou classes - sont définies en fonction de variables dont le choix dépend du problème à résoudre. Lorsque la stratification d'une photographie ou d'un groupe de photographies aura été faite, il suffira d'appliquer une méthode d'échantillonnage statistique appropriée pour obtenir le résultat cherché.

Une illustration particulièrement nette de cette méthode nous sera fournie par l'étude de l'inventaire volumétrique d'un massif foreslier.

Soit à réaliser l'inventaire d'un massif forestier inconnu ou peu connu, avec des possibilités d'investigation au sol réduites et dans des conditions de temps, d'accessibilité et de crédits rendạnt ịmpos- 
sible un recensement complet sur le terrain. Les phases de l'opération seront les suivantes:

$\mathrm{I}^{\circ}$ Détermination des classes forestières. - Un examen rapide des photographies permettra de choisir parmi les variables possibles celles qu'il convient d'utiliser pour l'identification des classes:

Ces variables peuvent être:

a) l'essence forestière;

b) la hauteur des arbres;

c) les dimensions de la couronne;

d) la densité du peuplement ;

e) la structure du peuplement (mode de traitement);

f) les conditions stationnelles (altitude, exposition, nature, profondeur ou humidité du sol).

Les combinaisons $a b d, a b e, b a d, b d f$ pourront fournir dans la plupart des cas des indications suffisantes.

Les classes pourront être délimitées au crayon dermographique sur les photographies elles-mêmes observées sous le stéréoscope, puis reportées suivant le cas, soit sur une carte, soit sur un calque ou un canevas, à vue ou après restitution sommaire s'il y a lieu.

$2^{\circ}$ Echantillonnagc. - I.e choix de la méthode d'échantillonnage - systématique, sélectif, au hasard -, ainsi que le nombre d'unités d'échantillonnage à définir, dépendent des conditions de l'expérimentation et en particulier de la qualité des photographies, de la nature et de la complexité des peuplements, des possibilités d'opérations au sol, de la précision recherchée.

Dans le cas considéré, on choisira, par exemple, des unités d'échantillonnage d'une surface de 0,25 ha, régulièrement réparties selon les mailles d'un réseau dont la densité pourra être variable suivant les classes, en fonction de la nature des peuplements et de l'importance du volume de chacun d'eux. En outre, l'implantation de ce réseau pourra tenir compte des possibilités ultérieures d'opérations ou sol (Cf. infra $3^{\circ}$ ).

Dans tous les cas, il sera utile de définir ces unités au moyen d'une grille-repère permettant de préciser sans ambiguité les coordonnées de chaque unité d'échantillonnage sur tune photographie.

$3^{\circ}$ Estimation du volume. - Il y a lieu d'envisager ici deux cas, selon que les opérations au sol seront possibles ou difficiles:

a) Dans le premier cas, le nombre d'unités d'échantillonnage peut être assez réduit: on évaluera au sol, avec la plus grande précision possible, le volume des unités qui auront été retenues pour chaque classe, et connaissant la surface de ces classes (appréciable au planimètre ou au calque millimétré), on en déduira aișément le volume de chacune d'elles. 
b) S'il est impossible d'envisager des opérations au sol, l'estimation volumétrique devra être totalement photogrammétrique: il est alors nécessaire de retenir pour chaque classe un nombre plus élevé d'unités d'échantillonnage, et de disposer de tables permettant de calculer, pour une essence déterminée, les volumes en fonction des variables identifiables sur photographies.

Sur le plan pratique, l'efficacité de cette méthode d'inventaire volumétrique dépend, dans une large mesure, de la mise au point correcte du matériel de référence mis à la disposition des interprétateurs, en vue d'une évaluation commode des variables. Un jeu de plaques transparentes, utilisables sous le stéréoscope, et portant gravées les indications nécessaires, permettra d'apprécier rapidement:

- le diamètre des couronnes,

- la densité des peuplements,

- la hauteur des arbres en fonction des différences de parallaxe et de l'échelle,

- la surface des unités d'échantillonnage en fonction de l'échelle.

Quant aux tables volumétriques, leur mise au point doit être faite dans des massifs forestiers témoins où l'on puisse disposer à la fois de renseignements recueillis au sol et de missions photographiques contemporaines.

A titre d'exemple, voici l'une des méthodes décrite par GARver et Moessner, utilisées par le Service Forestier des Etats-Unis - Central States Forest Experiment Station - :

Les variables sont: le diamètre des couronnes, la densité du couvert, la hauteur des arbres, combinaison qui s'est avérée la meilleure, après contrôle au sol.

Les interprétateurs disposent des gabarits transparents suivants:

I. - Un gabarit d'échelles permettant d'apprécier rapidement l'échelle de chaque photographie et les surfaces des unités d'échantillonnage à 'étudier.

2. - Un gabarit de diamètres des couronnes: points noirs de diamètre croissant permettant d'apprécier, grâce à une table de conversion en fonction de l'échelle des photographies, le diamètre des couronnes (de 5 pieds en 5 pieds), dans une unité.

3. - Un gabarit de densité du peuplement, donnant, pour une échelle déterminée, les pourcentages de couvert forestier (série de carrés à l'intérieur desquels sont gravés des points noirs de plus en plus nombreux).

4. - Une règle parallaxe, graduée en millièmes de pouces et permettant d'évaluer les différences de parallaxe entre le " toit ) d'un massif forestier (ou le sommet d'un arbre) et le sol, et d'en déduire, grâce à une table de conversion, la hauteur en fonction de l'échelle.

Les indications recueillies dans une unité d'échantillonnage, grâce 
à ces transparents, sont consignées sur une fiche, ainsi que divers autres renseignements relatifs à la station: physionomie ou structure du peuplement, type de sol ou de (" site », etc...

Chaque fiche reçoit enfin l'indication du volume défini d'après des tables volumétriques qui donnent, pour chaque classe de couronne (de 5 en 5 pieds), le volume à l'acre en fonction de la densité du couvert (de Io en Io \%, entre 5 et $95 \%$ ) et la hauteur moyenne des arbres (de 5 en 5 pieds).

Il est certain que c'est la mise au point de ces tables qui constitue le problème le plus délicat: le mieux serait de disposer de tables établies pour chaque essence ou chaque type de peuplement, mais de l'avis des interprétateurs américains, une table ( passe-partout ) peut permettre des estimations acceptables, dans la région pour laquelle elle a été établie, surtout s'il est possible de préciser les résultats par quelques sondages de contrôle.

D'après Pope, c'est la combinaison des variables: hauteur totale moyenne - densité du couvert - diamètre moyen des couronnes, qui fournit les meilleurs résultats: au cours d'expériences faites sur des peuplements de Douglas, les estimations photogrammétriques, conduites d'après ce principe, ont donné des résultats différant de moins de ro \% (quelquefois de moins de $3 \%$ ) des chiffres recueillis an sol.

\section{IV. - Possibilités et Limites de L'INTERPRÉtation PHOTOGRAPIHIQUE EN MATIÈRE FORESTIÈRE}

Les applications forestières de la photographie aérienne sont multiples: la plupart des missions incombant à un Ingénieur forestier (relatives aussi bien à la sylviculture qu'à la restauration ou la conservation du sol, au sens le plus large du terme) peuvent être facilitées, sinon parfois totalement résolues, grâce à l'utilisation des photographies aériennes.

Toutefois, les techniques photogrammétriques n'auront de véritable efficacité que si elles sont correctement liées à des opérations an sol selon les quatre phases de l'interprétation définies plus haut (Cf. I, $4^{\circ}$ ), c'est par une coordination constante des deux méthodes que l'on obtiendra les résultats les meilleurs.

En effet, si la photographie aérienne permet, dans une très large mesure, d'orienter, de limiter ou de compléter les travaux sur le terrain, elle ne saurait les supprimer totalement, si ce n'est pour certaines études de recensement physionomique ou de cartographie relativement sommaire. Par contre, sous condition d'opérations au sol le plus souvent très simples et localisées, mais toujours dirigées par des impératifs (" photogrammétriques ), l'interprétation des photographies peut être poussée très avant.

Il y a cependant des limites à ces possibilités d'investigation :

- l'interprétation est limitée à l'observation du toit des objets, ce 
qui exclut toute étude des éléments sous-jacents, bien (qu'on puisse parfois déceler des corrélations entre ces éléments et certains critères photogrammétriques;

- les ombres portées (surtout en montagne), la neige, les nuages, sont autant de lacunes de documentation;

- l'échelle des photographies n'est pas toujours suffisante pour permettre certaines observations (particulièrement pour l'identification spécifique); cependant l'intérêt des photographies à grande échelle est rapidement limité par leur prix de revient et l'absence de repères: pour de multiples raisons l'échelle moyenne idéale est le I/I2'000I / 5000 ;

- il faut enfin tenir compte des similitudes dans l'aspect photographique d'objets différents (similitudes d'autant plus fréquentes que l'échelle est plus petite) et de la variabilité des images d'un même objet suivant les conditions de prise de vues, la saison, l'éclairement, l'émulsion des clichés, etc...

\section{Conclusion}

C'est donc dans une exacte appréciation des possibilités de la photographie aérienne et des conditions de travail sur le terrain que peut être désormais étudiée l'écononic de toute opération impliquant, d'une manière quelconque, la connaissance du milieu naturel sous ses divers aspects.

Entre certains problèmes où le recours à la photographic est un luxe - ou une illusion - , ct ceux qu'elle s'avère seule capable de résoudre, il y a place pour une multitude de cas où elle constitue un appoint précieux ou indispensable.

Il convient cependant de ne jamais oublier, surtout lorsque. à priori, la balance ne semble pas pencher en sa faveur, que la photographie aérienne offre avant toute chose un incomparable potentiel de synthèse. Elle fixc, dans un " instantané » que le stéréoscope rend plus vivant, une vision coordonnée et totale des éléments de nombreux problèmes, que de laborieuses opérations au sol permettent sans doute d'éclairer, mais pas toujours de résoudre.

Paul Rey, Agrégé de l'Université, Directeur technique du Service de la Carte de la Végétation. 